
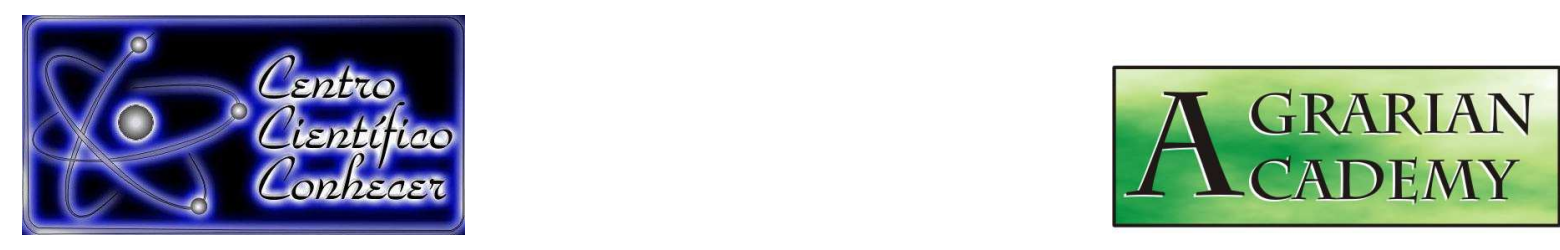

\title{
LEVANTAMENTO DA INCIDÊNCIA DE PRAGAS E DOENÇAS NA CULTURA DA LARANJA (Citrus sinensis L.) NA COMUNIDADE DO CUBITEUA, CAPITÃO POÇO/PA
}

\author{
Luane Laíse Oliveira Ribeiro ${ }^{1}$, Letícia do Socorro Cunha ${ }^{2}$, Leidiane de Oliveira \\ Lima $^{3}$, Wanderson Cunha Pereira ${ }^{4}$
}

1 Graduanda do Curso de Agronomia da Universidade Federal Rural da Amazônia (luanelaiseifpa@hotmail.com) Capitão Poço-Brasil

2 Graduanda do Curso de Agronomia da Universidade Federal Rural da Amazônia Campus Capitão Poço-Brasil

3 Graduanda do Curso de Agronomia da Universidade Federal Rural da Amazônia Campus Capitão Poço-Brasil

4 Professor Mestre da Universidade Federal Rural da Amazônia Capitão PoçoBrasil

Recebido em: 30/11/2017 - Aprovado em: 15/12/2017 - Publicado em: 31/12/2017 DOI: 10.18677/Agrarian Academy 2017b18

\begin{abstract}
O município de Capitão Poço, localizado no Nordeste Paraense, destaca-se como um dos principais produtores de laranja do Estado do Pará. A cultura dos citros é alvo constante de inúmeras pragas e doenças, que, encontrando condições favoráveis ao seu desenvolvimento, são capazes de causar danos irreversíveis. Neste sentido, o trabalho teve como objetivo avaliar as pragas e doenças de maior incidência dentre quatro mais comuns no cultivo de laranja Pêra (Citrus sinensis (L.) Osbeck) na localidade do Cubiteua, município de Capitão Poço/PA. As avaliações foram realizadas em campo, por meio de diagnose visual, em cinco propriedades distintas produtoras de laranja, onde foram escolhidas 100 plantas para avaliação de cada pomar. Constatou-se que a praga de maior ocorrência foi a larva minadora, sendo está presente em todos as propriedades de laranja estudadas, atingindo o valor máximo de $100 \%$, causando redução de área foliar e queda prematura de folhas. Encontrou-se também, a presença da escama farinha em todos os pomares, porém o que apresentou maior incidência foi o laranjal P5 com 3.067\% a mais que o laranjal P2. A doença que apresentou maior incidência foi a mancha graxa, presente em todas as propriedades atingindo o valor de $100 \%$. Com relação a clorose variegada dos citros (CVC), a maior incidência foi no laranjal P1 com a porcentagem de $567 \%$ a mais que o P4. Foi possível conhecer a principal praga e doença dos pomares avaliados, entender o nível de danos que estas causam, bem como a forma de controle que os agricultores adotam.
\end{abstract}

RESUMO

PALAVRAS-CHAVE: citros, diagnose, pomar 


\title{
SURVEY OF THE INCIDENCE OF PESTS AND DISEASES IN ORANGE CULTURE (Citrus sinensis L.) IN THE CUBITEUA COMMUNITY, CAPITAO POÇO / PA
}

\begin{abstract}
The municipality of Capitão Poço, located in the Northeast of Paraense, stands out as one of the main orange producers in the state of Pará. Citrus is a constant target of numerous pests and diseases, which, by finding favorable conditions for its development, are capable cause irreversible damage. In this sense, the objective of this work was to evaluate the most common pests and diseases among the four most common pests (Citrus sinensis (L.) Osbeck) in the locality of Cubiteua, municipality of Capitão Poço / PA. The evaluations were performed in the field, through visual diagnosis, in five different orange producing properties, where 100 plants were selected for evaluation of each orchard. It was verified that the pest of greater occurrence was the larvae, being present in all the properties of orange studied, reaching the maximum value of $100 \%$, causing reduction of leaf area and premature fall of leaves. It was also found the presence of flour scale in all orchards, but the one with the highest incidence was P5 oranges with 3.067\% more than P2 oranges. The disease that presented the highest incidence was the spot grease, present in all properties reaching the value of $100 \%$. Regarding citrus variegated chlorosis (CVC), its highest incidence was in the P1 orange tree, with a percentage of $567 \%$ higher than P4. It was possible to know the main pest and disease of the evaluated orchards, to understand the level of damages that they cause, and the form of control that the farmers adopt.
\end{abstract}

KEYWORDS: Citrus, diagnosis, orchard

\section{INTRODUÇÃO}

Citros é considerado um grupo de fruteiras importante no Brasil, não somente pela qualidade nutritiva dos frutos, mas também devido à função social e econômica que exerce como produto de exportação (OLIVEIRA et al., 2012a). O United States Department of Agriculture-USDA (2017) no relatório divulgado no mês janeiro estimou para a safra mundial de laranja in natura - 2016/17, uma produção de 49.610 mil toneladas. Segundo aquele Departamento, a produção deverá ser (+) $5,39 \%$, em comparação ao ano anterior. O Estado do Pará é responsável por 1,02\% (258.758 toneladas) da produção de laranja no Brasil, desse montante o município de Capitão Poço é responsável por 57\% (146.370 toneladas) do total produzido pelo estado (IBGE, 2015).

O município de Capitão Poço, localizado no Nordeste Paraense, destaca-se como um dos principais produtores de laranja do Estado, sendo a hortifruticultura importante dentro de uma perspectiva social, econômica e ecológica dada as condições favoráveis de clima, solo e mercado, características essas que proporcionam aumento considerável no cultivo de laranja no município, que em 2011 apresentava cerca de dois milhões de plantas, tornando-se maior produtor de citros da região Norte do Brasil (IBGE, 2011).

A cultura dos citros é alvo constante de inúmeras pragas e doenças, que, encontrando condições favoráveis ao desenvolvimento, são capazes de causar danos irreversíveis. A quantidade e a qualidade das frutas cítricas são frequentemente ameaçadas devido aos danos provocados à cultura que, dependendo da intensidade do ataque, pode torná-la improdutiva ou levar à erradicação (FUNDO DE DEFESA DA CITRICULTURA, 2008). Devido a importância 
que essa cultura representa para a agricultura brasileira, problemas relacionados a fitossanidade ocasionados por pragas e doenças, podem limitar o setor citrícola, causando grandes prejuízos, o que justifica os altos investimentos para o controle fitossanitário da cultura (OLIVEIRA et al., 2012b).

$\mathrm{O}$ aparecimento de pragas e doenças nas lavouras citrícolas mediou o desenvolvimento de pesquisas em citrus e a adoção de novas técnicas e tecnologias de produção que asseguraram a competitividade produtiva do setor. Apesar da importância das pesquisas fitossanitárias para o desenvolvimento tecnológico da produção e dos avanços nas pesquisas de fitopatologia de citrus, não há solução curativa para as doenças na citricultura, por isso o controle baseia-se primordialmente na adoção de medidas de manejo baseadas em inspeções de plantas, erradicações e pulverizações com agroquímicos (BARBOSA; GITAHY, 2016).

É de conhecimento de todos os agricultores, técnicos e dos órgãos competentes a existência do ataque fitossanitário na citricultura local. Entretanto, pouco se sabe sobre a real infestação e distribuição de várias pragas e doenças na comunidade do Cubiteua/PA, devido ao conhecimento científico limitado sobre a incidência dos mesmos. Neste sentido é de fundamental importância que pesquisas voltadas ao levantamento das espécies sejam intensificadas e quantificadas para promover maior compreensão sobre o assunto estudado bem como conhecer o potencial de danos causados nesta localidade. Neste sentido, o presente trabalho teve como objetivo verificar as pragas e doenças de maior incidência dentre quatro mais comuns no cultivo de laranja Pêra (Citrus sinensis (L.) Osbeck) na localidade do Cubiteua no município de Capitão Poço/PA.

\section{MATERIAL E MÉTODOS}

A pesquisa foi realizada na zona rural, mais especificamente na localidade do Cubiteua no município de Capitão Poço, localizada na latitude 014ㄴ'47" S e

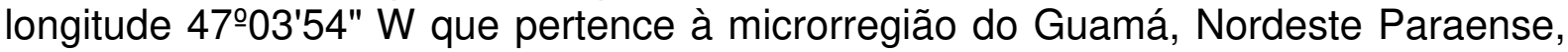
no período de 27 de agosto de 2015 a 06 de janeiro de 2016. O clima da região é do tipo Am (LA distrófico), com precipitação anual de $2250 \mathrm{~mm}$, e uma altitude de $71 \mathrm{~m}$ e temperatura média de $25^{\circ} \mathrm{C}$ (INMET, 2015).

As avaliações foram realizadas em campo, por meio de diagnose visual, em cinco propriedades distintas produtoras de laranja: Pomar 1 (P1), Pomar 2 (P2), Pomar 3 (P3), Pomar 4 (P4) e Pomar 5 (P5), com idades de plantio de quatro a sete anos, onde foram levantadas 100 amostras aleatórias de cada pomar. Todos os pomares são cultivados com a variedade Pêra Rio (Citrus sinensis (L.) Osbeck), enxertadas em limão cravo (Citrus limonia (L.) Osbeck).

O estudo teve o intuito de saber a incidência de pragas e doenças que geralmente são encontradas nos pomares da região e causam dano econômico na cultura se não forem controladas, sendo as pragas: ortézia (Orthezia praelonga), pulgão preto (Toxoptera citricidus), escama farinha (Pinnaspis aspiditrae), larva minadora (Phyllocnistis citrella) e doenças: clorose variegada dos citros (CVC) (Xilella fastidiosa), fumagina (Capnodium citri), mancha aureolada (Pelicularea filamentosa) e mancha graxa (Mycosphaerella citri).

O levantamento feito nos pomares foi com o auxílio de uma planilha sendo atribuída a presença ou ausência com número 1 e 0 respectivamente das pragas e doenças para cada sistema de produção. A planta foi observada no todo, com atenção especial para as regiões mais atacadas pelas pragas. Posteriormente os 
dados foram tabulados e esboçados em gráfico com o auxílio da planilha Microsoft Excel e em seguida foram expostos em percentual para fazer a comparação das propriedades em relação as pragas e doenças, de forma a facilitar o entendimento.

\section{RESULTADOS E DISCUSSÃO}

Os resultados obtidos, referentes à ocorrência das pragas nos pomares dos produtores da comunidade do Cubiteua/PA encontram-se expostos na Figura 1. Com base nos cinco pomares de laranja avaliados, constatou-se que a praga de maior ocorrência foi a larva minadora (Phyllocnistis citrella), sendo esta presente em todas as propriedades de laranja estudadas, atingindo o valor máximo de $100 \%$ de plantas com sintomas do ataque, que por sua vez causa a redução da área foliar e queda prematura das folhas. No estudo feito por Ferreira et al., (2013) também foi constado que a larva minadora dos citros é a praga com maior incidência nos pomares de laranja lima de Santana do Mundaú-AL. Todos os pomares avaliados apresentavam $100 \%$ de plantas com sintomas de ataque da praga sobre as folhas.

No que se refere ao índice de pragas nos pomares, constatou-se que no laranjal P1, a ortézia (Orthezia praelonga) apresentou maior incidência de plantas afetadas com uma porcentagem de $1.150 \%$ a mais quando comparada ao P2, sendo que não foi encontrada a ocorrência de ortézia no P3.

A ortézia suga a seiva, provoca a murcha, queda de folhas e frutos, diminui a quantidade de açúcares e ácidos, resultando nos chamados 'frutos aguados' e ainda provoca o aparecimento da fumagina (fungo preto) que recobre folhas, frutos e ramos atacados. Em altas populações pode causar morte da planta (CARVALHO; MACEDO, 2015).

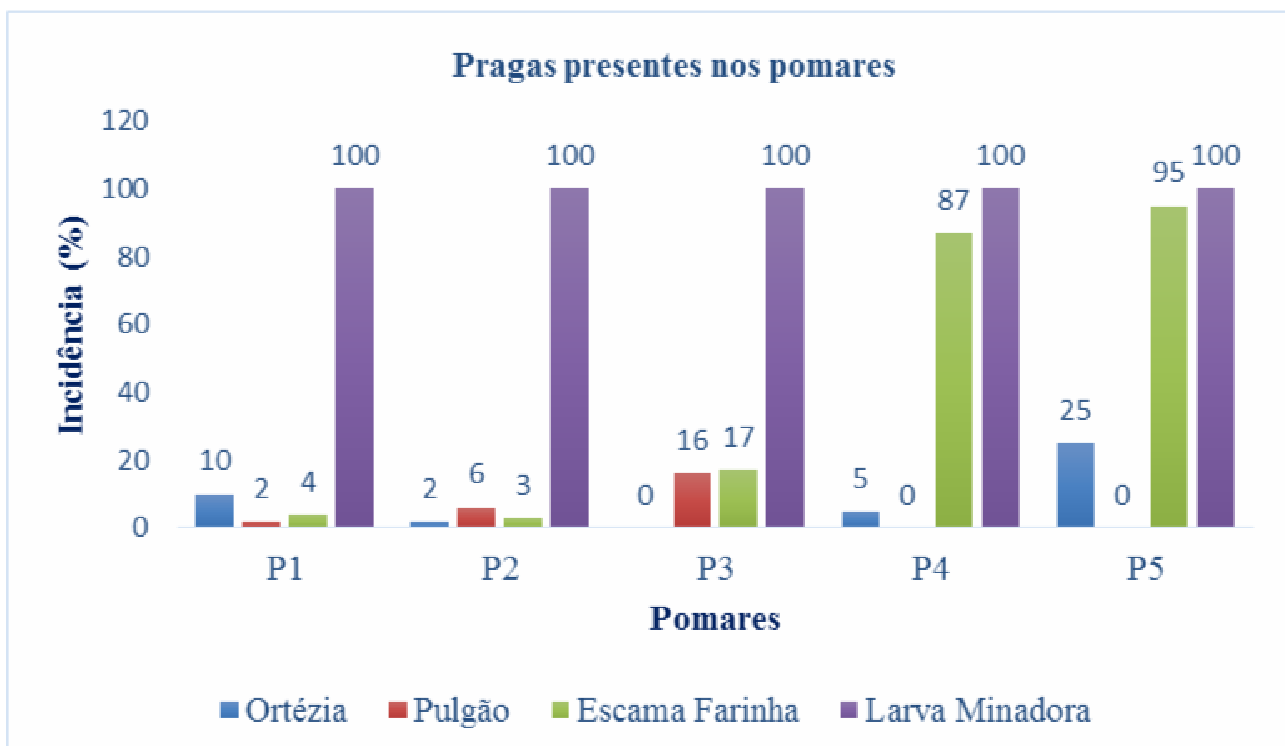

FIGURA 1 Ocorrência das Pragas nos cinco pomares produtores de laranja na comunidade do Cubiteua Capitão poço/PA.

Fonte: Autores (2017)

Segundo os resultados obtidos, observou-se que o pulgão (Toxoptera citricidus) está frequente em $700 \%$ do laranjal P3, sendo esta ocorrência superior em relação ao P1. Foi possível constatar a ausência desta praga em dois pomares, sendo: P4 e P5. Os pulgões vivem em colônias e, devido ao ataque intenso e ao ato de introduzir as peças do aparelho bucal no tecido vegetal, produzem deformações AGRARIAN ACADEMY, Centro Científico Conhecer - Goiânia, v.4, n.8; p.178 2017 
nas folhas (encarquilhamento). Os maiores danos são provocados em plantas novas, quando atacam brotações, folhas em desenvolvimento e botões florais (CARVALHO; MACEDO, 2015).

Encontrou-se também, a presença da escama-farinha (Pinnaspis aspiditrae) em todos os pomares visitados, porém o que apresentou maior incidência foi o laranjal P5 com 3.067\% a mais em relação ao laranjal P2. De acordo com Ferreira et al. (2013), a escama-farinha também está presente em todos os pomares de laranja lima avaliados em Santana do Mundaú-AL, com incidência variando de 74 a $98 \%$ das plantas. A escama-farinha pode causar prejuízos aos citricultores de laranja Pêra na comunidade estudada, pois trata-se de uma praga que suga a seiva da planta, injeta toxinas e expele um líquido açucarado, que, caindo sobre a planta, favorece o desenvolvimento de fungo do gênero Capnodium, que forma uma camada preta, conhecida como fumagina (BARBOSA et al., 2007).

Os resultados referentes à ocorrência das doenças nos pomares de laranja na comunidade do Cubiteua encontram-se na Figura 2. Observou-se que dentre os cinco pomares avaliados, a doença que apresentou maior incidência foi a mancha graxa (Mycosphaerella citri), presente em todas as propriedades atingindo o valor máximo de $100 \%$.

Os sintomas são encontrados em folhas de todas as cultivares comerciais, sendo mais preocupantes em pomelos, limões e laranjeiras-doces precoces, enquanto a laranjeira 'Valência' e as tangerineiras são mais resistentes à manchagraxa. São identificadas manchas escuras ou pretas, lisas, brilhantes e com aspecto de graxa na face inferior das folhas, com manchas amarelas correspondentes na face superior apenas no início do desenvolvimento da doença. As folhas doentes podem cair antes da completa manifestação da doença. Os sintomas em frutos aparecem após três a seis meses, na forma de lesões muito pequenas, necróticas. A casca do fruto pode adquirir cor marrom ou preta, podendo os sintomas serem confundidos com os causados pelo ácaro-da-falsa-ferrugem (KOOLLER, 2013).

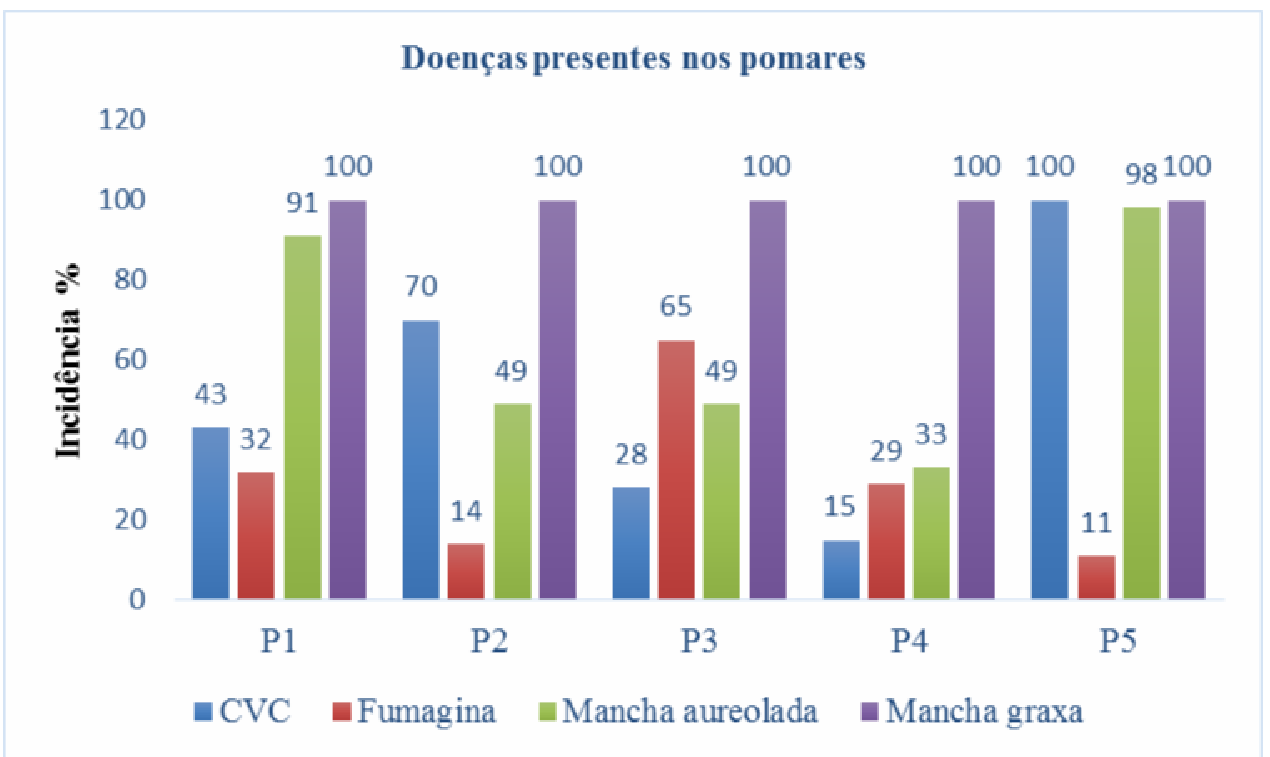

FIGURA 2 Ocorrência das doenças nos cinco pomares produtores de laranja na comunidade do Cubiteua Capitão $\mathrm{Poço/Pa.}$

Fonte: Autores (2017) 
Com relação à doença clorose variegada dos citros (CVC), a maior incidência foi no laranjal P1 com a porcentagem de $567 \%$ a mais que o $\mathrm{P} 4$, sendo que este apresenta menor ocorrência da doença, quando comparadas com as demais propriedades. Segundo Filho et al. (2016) a CVC é uma doença causada por uma bactéria, que causa manchas amareladas e variegadas nas folhas dos citros; secamento de ramos, amarelecimento precoce, endurecimento e redução no tamanho dos frutos.

Quanto à fumagina (Capnodium citrı), pode-se constatar que a propriedade que apresentou maior índice da doença foi o laranjal P3, onde ocorreu um percentual de $491 \%$ a mais que na propriedade P5. Mesmo em baixa infestação, a doença possui potencial para causar danos econômicos aos agricultores, pois espalha-se na forma de uma crosta espessa e negra cobrindo total ou parcialmente toda a área da folha e em alguns casos também os frutos, prejudicando a planta por reduzir à área fotossintética, a respiração, a transpiração e por tornar os frutos com aparência pouco aceitável no mercado. Geralmente, formigas, cochonilhas e pulgões estão associados à presença de fumagina nas plantas (LEMOS FILHO; PAIVA, 2006).

Comparando os pomares que apresentaram maior e menor presença da mancha aureolada (Pelicularea filamentosa), constatou-se que o pomar P5 possui uma porcentagem de $197 \%$ a mais que o pomar P4. Os resultados observados por Silva et al. (2009), mostram que esta doença é considerada de menor importância, e têm sido pouco estudada, no entanto, ainda segundo estes autores e também Melo e Andrade (2006), a presença constante da doença nas regiões Norte e Nordeste do Brasil, causa intensa desfolha principalmente no período chuvoso.

\section{CONCLUSÕES}

Diante do estudo foi possível conhecer a principal praga e doença de maior incidência nos pomares avaliados bem como entender o nível de dano que estas podem causar caso não sejam controladas. Os agricultores por sua vez conseguem conviver com estes fitopatógenos e quando não, utilizam produtos químicos que minimizem o ataque causado nos pomares. Porém é perceptível a falta de assistência técnica que possa auxiliá-los no combate a essas pragas e doenças, e que potencialize a produção citrícola da comunidade e do município como um todo.

\section{AGRADECIMENTOS}

Ao Prof. Msc. Wanderson Cunha Pereira pela orientação no desenvolvimento e construção deste trabalho, e em especial aos agricultores da comunidade do Cubiteua pela contribuição e aceitação para realização da pesquisa em suas propriedades.

\section{REFERÊNCIAS}

BARBOSA, F. R.; NETO, L. G.; CARVALHO, G. K. L.; CARVALHO, R. S. Manejo e controle da cochonilha ortézia (Orthezia praelonga), em plantios irrigados de acerola, no submédio São Francisco. Petrolina, PE: Embrapa Semi-Àrido, 2007. P. 02. (Embrapa Semi-Àrido. Circular técnico, 83).

BARBOSA, G. R.; GITAHY, L. Pragas e doenças: controvérsias na constituição da cadeia produtiva da laranja no Estado de São Paulo dos anos 1930 aos 2000. Desafio Online, Campo Grade, v. 4, n. 2, agosto. 2016. Disponível em: http://www.desafioonline.ufms.br/index.php/deson/article/view/2084/1339

AGRARIAN ACADEMY, Centro Científico Conhecer - Goiânia, v.4, n.8; p.180 2017 
CARVALHO, R. S.; MACEDO, L. R. Guia para: reconhecimento dos principais insetos e ácaros praga e inimigos naturais em citros. Cruz das Almas: Embrapa Mandioca e Fruticultura BA., 2015.

FERREIRA, J. T. P.; FERREIRA, E. P.; SILVA, W. C.; MONTEIRO, J. H. A.; ROCHA, I. T. M. et al. Estudo fitossanitário em pomares de laranja lima (citrus sinensis (L.) Osb.) em Santana do Mundaú - AL. Agropecuária Científica no Semiárido, Alagoas, v.9, n. 1, p. 62-61, jan/mar, 2013. Disponível em: http://revistas.ufcg.edu.br/acsa/index.php/ACSA/article/download/265/pdf

FILHO, H. P. S.; OLIVEIRA, A. A. R.; HADDAD, F. Controle alternativo das doenças dos citros. Cruz das Almas: Embrapa Mandioca e Fruticultura BA, 2016. p. 13.

FUNDO DE DEFESA DA CITRICULTURA. 2008. Disponível em: $<\mathrm{http}: / /$ www.fundecitrus.com.br/doenca/moscas_negra.html>. Acesso em: 25 fev. 2008.

IBGE - Instituto Brasileiro de Geografia e Estatística. Pesquisa agrícola municipal. Disponível em: <http:// www.ibge.gov.br>. Acesso em: 08 março de 2015.

IBGE - Instituto Brasileiro de Geografia e Estatística. Indicadores IBGE. Estatística da Produção Agrícola (2011). Disponível em: $<$ http://www.ibge.gov.br/home/estatistica/indicadores/agropecuaria/lspa/estProdAgr_ 201112.pdf>. Acesso: 31/01/2012.

INMET (BRASIL) - Instituto Nacional de Meteorologia. Disponível em: http://www.inmet.gov.br/ Acesso em: 20 de jan. 2015.

KOLLER, O.L. (Org.) Citricultura catarinense. Florianópolis: Epagri, 2013. 319p.

LEMOS FILHO, J.P.; PAIVA, É. A. S. The effects of sooty mold on photosynthesis and mesophyll structure of mahogany (Swietenia macrophylla king., meliaceae). Revista Bragantia, Campinas, v.65, n.1, p.11-17, 2006. Disponível em: http://www.scielo.br/pdf/brag/v65n1/29033.pdf

MELO, M.B.; ANDRADE, L.N.T. Principais doenças da citricultura em Sergipe e seu controle. In: MELO, M.B.; SILVA, L.M.S. Aspectos técnicos dos citros em Sergipe. Aracaju: EMBRAPA Tabuleiros Costeiros; Deagro, p. 71-84, 2006.

OLIVEIRA, F. J.; FARIAS, P. R. S.; SILVA, A. G.; RODRIGUES, K. C. V.; ARAÚJO, F. C. B. Uso da geoestatística para avaliação da distribuição espacial da leprose dos citros. In: Seminário Anual de Iniciação Científica, 10., 2012a, Belém. Anais...Belém: Universidade Federal Rural da Amazônia, 2012.

OLIVEIRA, I. P.; OLIVEIRA, L. C.; MOURA, C. S. F. T. Alguns fatores bióticos e abióticos que afetam a qualidade dos produtos da laranja no mercado. Revista Faculdade Montes Belos, v. 5, n. 4, agosto. 2012b. Disponível em: revista.fmb.edu.br/index.php/fmb/article/download/91/86

SILVA, S.X.B.; LARANJEIRA, F.F.; SOARES, A.C.F.; MICHEREFF, S.J. Amostragem, caracterização de sintomas e escala diagramática da mancha graxa 
dos citros (Mycosphaerella citri) no Recôncavo Baiano. Ciência Rural, v.39, n.3, p.896-899, 2009. Disponível em: http://dx.doi.org/10.1590/S010384782008005000098

USDA - UNITED STATES DEPARTAMENT OF AGRICULTURE. Brazil citrus annual 2017. 2017. Disponível em: <http://www.conab.gov.br>. Acesso em: 07 jul 2017. 